\title{
FLOW INSTABILITY THROUGH A CURVED DUCT WITH SQUARE CROSS SECTION
}

\author{
${ }^{1}$ Rabindra Nath Mondal, ${ }^{2}$ Md. Saidul Islam and ${ }^{3}$ Shah Md. Tarif Hossain \\ ${ }^{1,2)}$ Mathematics Discipline; Science, Engineering and Technology School, \\ Khulna University, Khulna-9208, Bangladesh \\ Email: rnmondal71@yahoo.com \\ ${ }^{3)}$ Department of Mathematics, Govt. B. L. College, Khulna
}

Received 09.07.07 Accepted 27.06.09

\begin{abstract}
Flow instability through a curved duct with square cross section is numerically studied by using the spectral method over a wide range of the Dean number $0 \leq$ $D n \leq 5000$ for the curvature $\delta=0.1$. A temperature difference is applied between the vertical sidewalls for the Grashof number $G r=100$, where the outer wall is heated and the inner wall is cooled. After a comprehensive survey over the parametric ranges, two branches of asymmetric steady solutions are obtained by the Newton-Raphson iteration method. Linear stability of the steady solutions is then investigated. It is found that only the first branch is linearly stable in a couple of interval of $D n$ while the other branch is linearly unstable. Steady values of the Nusselt numbers, $\mathrm{Nu}$, are also calculated for two differentially heated vertical sidewalls. When there is no stable steady solution, time evolution of $\mathrm{Nu}$ is obtained and it is found in the unstable region the flow undergoes through various flow instabilities, if $D n$ is increased.
\end{abstract}

Key words: Curved square duct, Steady solutions, Time evolution, Dean number, Nusselt number

\section{Introduction}

The study of flow through a curved duct is of fundamental interest because of its numerous applications in fluid engineering, such as in heat exchangers, ventilators, gas turbines, aircraft intakes and centrifugal pumps. Blood flow in human veins and arteries is another important application of curved duct flow. The flow through a curved duct shows physically interesting features under the action of the centrifugal force caused by the curvature of the duct. The presence of curvature generates centrifugal forces which act at right angle to the main flow direction and produce secondary flows. Dean [1] was the first who formulated the problem in mathematical terms under the fully developed flow condition. He found the secondary flow consisting of a pair of counter rotating vortices caused by the centrifugal force. Since then, there have been a lot of theoretical and experimental works concerning this flow, the review articles by Berger et al. [2], Nandakumar and Masliyah [3] and Ito [4] may be referred. 
One of the interesting phenomena of the flow through a curved duct is the bifurcation of the flow because generally there exist many steady solutions due to channel curvature. Dennis and $\mathrm{Ng}$ [5], Nandakumar and Masliyah [6] and later Yanase, Gotoh and Yamamoto [7] studied dual solutions of the flow through a curved duct. Yang and Keller [8] studied the bifurcation of the flow for small curvature and found multiple branches of solutions. Thangam and Hur [9] studied the characteristics of laminar secondary flows in a curved rectangular duct. However, detailed bifurcation structure and linear stability of the steady solutions for fully developed flows in a curved square duct was investigated by Winters [10]. He applied bifurcation analysis to it and found that there are many symmetric and asymmetric steady solutions among which linearly stable ones are few. The existence of the multiple solutions of the flow through a curved duct with the large aspect ratio was first studied by Yanase and Nishiyama [11]. They obtained two kinds of solutions: the two-vortex solution and the four-vortex solution for the same aspect ratio. Yanase [12] gave a short comment that the solution oscillates temporally between the symmetric two- and four-vortex solutions for the Dean number at which no stable steady solution exists. Wang and Yang [13] performed a numerical study on fully developed bifurcation structure and stability of the forced convection in a curved square duct flow. In the succeeding paper, Wang and Yang [14] performed numerical as well as experimental investigations of periodic oscillations for the fully developed flow in a curved square duct. Flow visualization in the range of Dean numbers from 50 to 500 was conducted in their experiment. They showed, both experimentally and numerically, that a temporal oscillation takes place between symmetric/asymmetric 2-cell and 4-cell flows when there are no stable steady solutions.

One of the most important applications of curved duct flow may be the enhancement of the thermal exchange between two sidewalls, because it is possible that the secondary flow may convey heat and then increases the heat flux between two sidewalls. Chandratilleke and Nursubyakto [15] presented numerical calculations to describe the secondary flow characteristics in the flow through curved ducts of aspect ratios ranging from 1 to 8 that were heated on the outer wall, where they studied for small Dean numbers and compared the numerical results with their experimental data. Recently, Yanase et al. [16, 17] performed numerical investigations of isothermal and nonisothermal flows through a curved rectangular duct. They discussed the bifurcation structure of the solutions and studied the effect of secondary flows on convective heat transfer. Very recently, Mondal et al. [18, 19] performed numerical prediction of isothermal and non-isothermal flows through a curved duct of square cross section. They found a close relationship between unsteady solutions and the bifurcation diagram of steady solutions for the isothermal flow. They also investigated effects of curvature on the flow characteristics. However, complete bifurcation structure as well as effects of the Nusselt number on the flow characteristics were not shown in their paper for the nonisothermal flow in the curved square duct.

The objective of the present study is to understand the characteristics of non-isothermal flow through a curved square duct over a wide range of the Dean number by finding the steady solutions, investigating their linear stability and analyzing the nonlinear behavior of the unsteady solutions by time evolution calculation of the Nusselt number. 


\section{Governing Equations}

Consider a laminar flow of viscous incompressible fluid through a curved duct with square cross section whose width or height is $2 d$. The coordinate system with the relevant notation is shown in Fig. 1, where $\mathrm{C}$ is the center of the duct cross section, $\mathrm{O}$ the center of curvature and $L$ the radius of curvature. The $x-, y$ - and $z$-axes are taken to be in the horizontal, vertical and axial directions, respectively. It is assumed that the outer wall of the duct is heated while the inner one is cooled. The temperature of the outer wall is $T_{o}+\Delta T$ and that of the inner wall is $T_{o}-\Delta T$ where $\Delta T>0$. It is also assumed that the flow is uniform in the axial direction and driven by a constant pressure gradient $\mathrm{G}\left(G=-\frac{\partial P^{\prime}}{\partial Z^{\prime}}\right)$ along the center-line of the duct, i.e. the main flow in the $Z$-direction as shown in Fig. 1.

The variables are non-dimensionalized by using the representative length $d$ and the representative velocity $U_{0}=v / d$. We introduce the non-dimensional variables defined as

$$
\begin{aligned}
& u=\frac{u^{\prime}}{U_{0}}, v=\frac{v^{\prime}}{U_{0}}, w=\frac{\sqrt{2 \delta}}{U_{0}} w^{\prime}, \quad x=\frac{x^{\prime}}{d}, \quad y=\frac{y^{\prime}}{d}, \quad z=\frac{z^{\prime}}{d} \\
& T=\frac{T^{\prime}}{\Delta T}, \quad t=\frac{U_{0}}{d} t^{\prime}, \quad \delta=\frac{d}{L}, \quad P=\frac{P^{\prime}}{\rho U_{0}^{2}}
\end{aligned}
$$

where $u, v$, and $w$ are the non-dimensional velocity components in the $x, y$, and $z$ directions, respectively; $t$ is the non-dimensional time, $P$ the non-dimensional pressure, $\delta$ the non-dimensional curvature, and temperature is non-dimensionalized by $\Delta T$. Henceforth, all the variables are nondimensionalized if not specified.

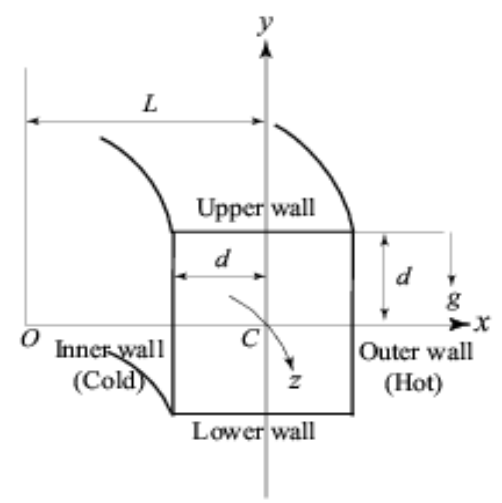

Figure 1: Coordinate system.

Since the flow field is uniform in the $Z$-direction, the sectional stream function $\psi$ is introduced as

$$
u=\frac{1}{1+\delta x} \frac{\partial \psi}{\partial y}, v=-\frac{1}{1+\delta x} \frac{\partial \psi}{\partial x} .
$$


The basic equations for $w, \psi$ and $T$ are derived from the Navier-Stokes equations and the energy equation under the Boussinesq approximation as

$$
\begin{aligned}
(1+\delta x) & \frac{\partial w}{\partial t}+\frac{\partial(w, \psi)}{\partial(x, y)}-D_{n}+\frac{\delta^{2} w}{1+\delta x}=(1+\delta x) \Delta_{2} w-\frac{\delta}{(1+\delta x)} \frac{\partial \psi}{\partial y} w+\delta \frac{\partial w}{\partial x} \\
& \left(\Delta_{2}-\frac{\delta}{1+\delta x} \frac{\partial}{\partial x}\right) \frac{\partial \psi}{\partial t}=-\frac{1}{(1+\delta x)} \frac{\partial\left(\Delta_{2} \psi, \psi\right)}{\partial(x, y)}+\frac{\delta}{(1+\delta x)^{2}} \\
& \times\left[\frac{\partial \psi}{\partial y}\left(2 \Delta_{2} \psi-\frac{3 \delta}{1+\delta x} \frac{\partial \psi}{\partial x}+\frac{\partial^{2} \psi}{\partial x^{2}}\right)-\frac{\partial \psi}{\partial x} \frac{\partial^{2} \psi}{\partial x \partial y}\right]+\frac{\delta}{(1+\delta x)^{2}} \\
& \times\left[3 \delta \frac{\delta^{2} \psi}{\partial x^{2}}-\frac{3 \delta^{2}}{1+\delta x} \frac{\partial \psi}{\partial x}\right]-\frac{2 \delta}{1+\delta x} \frac{\partial}{\partial x} \Delta_{2} \psi+w \frac{\partial w}{\partial y} \\
& +\Delta_{2}^{2} \psi-G r(1+\delta x) \frac{\partial T}{\partial x}, \\
& \frac{\partial T}{\partial t}+\frac{1}{(1+\delta x)} \frac{\partial(T, \psi)}{\partial(x, y)}=\frac{1}{\operatorname{Pr}}\left(\Delta_{2} T+\frac{\delta}{1+\delta x} \frac{\partial T}{\partial x}\right) .
\end{aligned}
$$

where

$$
\Delta_{2} \equiv \frac{\partial^{2}}{\partial x^{2}}+\frac{\partial^{2}}{\partial y^{2}}, \frac{\partial(f, g)}{\partial(x, y)} \equiv \frac{\partial f}{\partial x} \frac{\partial g}{\partial y}-\frac{\partial f}{\partial y} \frac{\partial g}{\partial x} .
$$

The Dean number Dn, the Grashof number $G r$ and the Prandtl number Pr, which appear in Eqs. (2) to (4) are defined as

$$
D n=\frac{G d^{3}}{\mu v} \sqrt{\frac{2 d}{L}}, \quad G_{r}=\frac{\beta g \Delta T d^{3}}{v^{2}}, \quad P_{r}=\frac{v}{k}
$$

where $\mu, \gamma, \kappa$ and $g$ are the viscosity, the coefficient of thermal expansion, the coefficient of thermal diffusivity and the gravitational acceleration respectively.

In the present study, the rigid boundary conditions for $w$ and $\psi$ are used as

$$
w( \pm 1, y)=w(x, \pm 1)=\psi( \pm 1, y)=\psi(x, \pm 1)=\frac{\partial \psi}{\partial x}( \pm 1, y)=\frac{\partial \psi}{\partial y}(x, \pm 1)=0
$$

and the temperature $T$ is assumed to be constant on the walls as

$$
T(1, y)=1, T(-1, y)=-1, T(x, \pm 1)=x .
$$

In this study, only $D n$ varies while $G r, \operatorname{Pr}$ and $\delta$ are fixed as $G r=100, \operatorname{Pr}=7.0$ (water) and $\delta=0.1$. 


\section{Numerical Method}

The method adopted in the present numerical calculation is the spectral method. By this method the variables are expanded in a series of functions consisting of the Chebyshev polynomials. That is, the expansion functions $\phi_{n}(x)$ and $\Psi_{n}(x)$ are expressed as

$$
\phi_{n}(x)=\left(1-x^{2}\right) C_{n}(x), \psi_{n}(x)=\left(1-x^{2}\right)^{2} C_{n}(x)
$$

where $C_{n}(x)=\cos \left(n \cos ^{-1}(x)\right)$ is the nth order Chebyshev polynomial. $w(x, y, t), \psi(x, y, t)$ and $T(x, y, t)$ are expanded in terms of $\phi_{n}(x)$ and $\psi_{n}(x)$ as

$$
\left.\begin{array}{c}
w(x, y, t)=\sum_{m=0}^{M} \sum_{n=0}^{N} w_{m n}(t) \phi_{m}(x) \phi_{n}(y), \\
\psi(x, y, t)=\sum_{m=0}^{M} \sum_{n=0}^{N} \psi_{m n}(t) \psi_{m}(x) \psi_{n}(y), \\
T(x, y, t)=\sum_{m=0}^{M} \sum_{n=0}^{N} T_{m n}(t) \phi_{m}(x) \phi_{n}(y)+x,
\end{array}\right\}
$$

where $M$ and $N$ are the truncation numbers in the $x$ - and $y$-directions respectively. The expansion coefficients $w_{m n}, \psi_{m n}$ and $T_{m n}$ are then substituted into the basic Eqs. (2), (3) and (4) and the collocation method is applied. As a result, nonlinear algebraic equations for $w_{m n}, \psi_{m n}$ and $T_{m n}$ are obtained. The collocation points are taken to be

$$
\left.\begin{array}{ll}
x_{i}=\cos \left[\pi\left(1-\frac{i}{M+2}\right)\right], & i=1, \ldots, M+1, \\
y_{i}=\cos \left[\pi\left(1-\frac{i}{N+2}\right)\right], & j=1, \ldots, N+1 .
\end{array}\right\}
$$

Where $i=1, \ldots, M+1$ and $j=1, \ldots, N+1$. The steady solutions are obtained by the NewtonRaphson iteration method and the convergence is assured by taking $\varepsilon_{p}<10^{-10}$, where subscript $p$ denotes the iteration number and $\varepsilon_{p}$ is defined as

$$
\varepsilon_{p}=\sum_{m=0}^{M} \sum_{n=0}^{N}\left[\left(w_{m n}^{(p+1)}-w_{m n}^{p}\right)^{2}+\left(\psi_{m n}^{(p+1)}-\psi_{m n}^{p}\right)^{2}+\left(T_{m n}^{(p+1)}-T_{m n}^{p}\right)^{2}\right] .
$$

In the present numerical calculations, $M=20$ and $N=20$ have been used for sufficient accuracy of the solutions (for details, see Mondal [20]).

To solve the steady solution, the time derivative terms $\partial w / \partial t, \partial \psi / \partial t$, and $\partial T / \partial t$ are taken to be zero and the expansion series (10) with coefficients $w_{m n}, \psi_{m n}$ and $T_{m n}$, being time independent, is substituted into the basic Eqs. (2) to (4). Finally, in order to calculate the unsteady solutions, the Crank-Nicolson and Adams-Bashforth methods together with the function expansion (10) and the collocation methods are applied to Eqs. (2) to (4). 


\section{Resistance Coefficient and the Nusselt Number}

In the present study, the resistance coefficient $\lambda$ is used as the representative quantity of the flow state. It is also called the hydraulic resistance coefficient, and is generally used in fluids engineering, defined as

$$
\frac{P_{1}^{*}-P^{*}{ }_{2}}{\Delta z^{*}}=\frac{\lambda}{4 d} \rho\left\langle w^{*}\right\rangle^{2}
$$

where quantities with an asterisk denote dimensional ones, \langle\rangle stands for the mean over the cross section of the duct and $\rho$ is the density. The mean axial velocity $\left\langle w^{*}\right\rangle$ is calculated by

$$
\left\langle w^{*}\right\rangle=\frac{v}{4 \sqrt{2 \delta d}} \int_{-1}^{1} d x \int_{-1}^{1} w(x, y, t) d y
$$

Since $\left(P_{1}^{*}-P_{2}^{*}\right) / \Delta z^{*}=G, \lambda$ is related to the mean non-dimensional axial velocity $\langle w\rangle$ as

$$
\lambda=\frac{4 \sqrt{2 \delta} D n}{\langle w\rangle^{2}}
$$

In the present study, the Nusselt number, $N u$, is defined as

$$
N u_{c}=\frac{1}{2} \int_{-1}^{1}\left[\frac{\partial T}{\partial x}\right]_{x=-1} d y, N u_{h}=\frac{1}{2} \int_{-1}^{1}\left[\frac{\partial T}{\partial x}\right]_{x=1} d y
$$

for steady solutions. For unsteady solutions, on the other hand, it is defined as

$$
N u_{T C}=\frac{1}{2} \int_{-1}^{1}\left\langle\left\langle\left.\frac{\partial T}{\partial x}\right|_{x=-1}\right\rangle\right\rangle d y, N u_{T h}=\frac{1}{2} \int_{-1}^{1}\left\langle\left\langle\left.\frac{\partial T}{\partial x}\right|_{x=1}\right\rangle\right\rangle d y
$$

where $\langle\langle\rangle\rangle$ denote an average over a time interval $\tau$. When the field is periodic, $\tau$ is taken as one period, and if it is chaotic $\tau$ is chosen to be an appropriate time interval.

\section{Results and Discussion}

\subsection{Solution structure}

We obtain two branches of asymmetric steady solutions over a wide range of the Dean number $0 \leq D n \leq 5000$ for $\delta=0.1$ and $G r=100$. The steady solution branches are obtained by the path continuation technique with different initial guesses as discussed in Mondal [20]. The bifurcation diagram is shown in Fig. 2 for $100 \leq D n \leq 2000$ using $\lambda$, the representative quantity of the solutions. The two steady solution branches are named as the first steady solution branch (first branch, thick solid line) and the second steady solution branch (second branch, thin solid line) respectively. In this regard, it should be remarked that for the isothermal flow in a curved square duct, Mondal et al. [18] obtained four branches of steady solutions with a bifurcating relationship among the branches, 
where two steady solutions are symmetric and two are asymmetric. For the nonisothermal flow in the present study, however, we obtain only asymmetric steady solutions, and it is found that there exists no bifurcating relationship between them in the parameter range investigated in this paper.

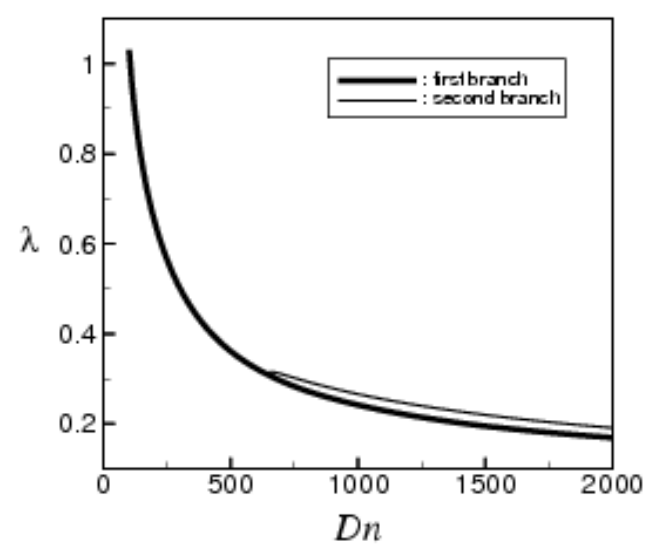

Figure 2: Steady solution branches for $G r=100$ and $100 \leq D n \leq 2000$ at $\delta=0.1$.

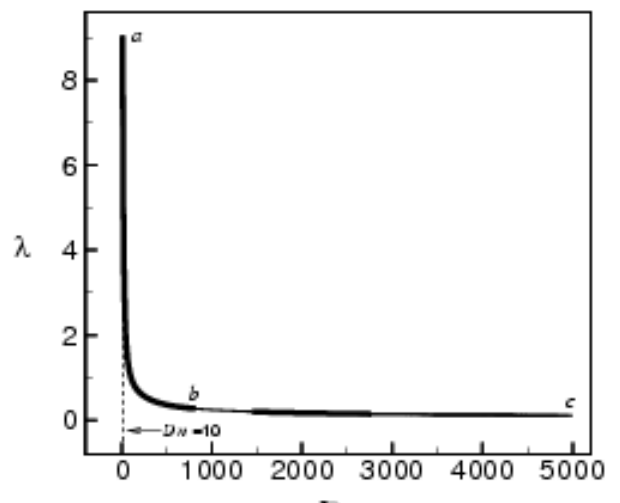

(a)

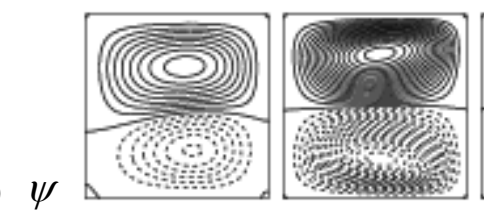

(b)

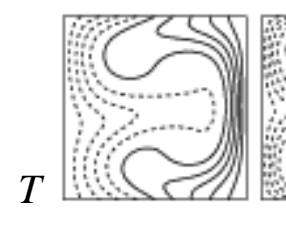

$D n=100(a)$

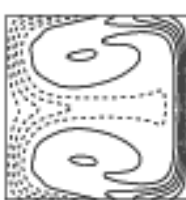

500
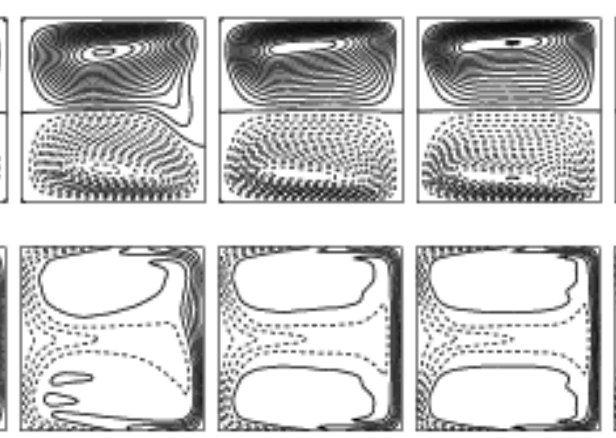

1000

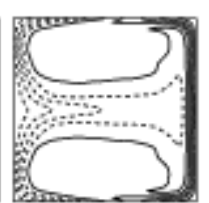

4000

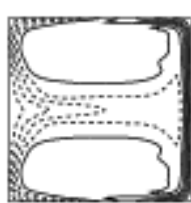

$5000(c)$

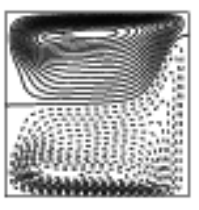

Figure 3: (a) First steady solution branch with the region of linear stability (bold line). (b) Contours of secondary flow (top) and temperature profile (bottom) on the first steady solution branch for $\mathrm{Dn}=$ $100(a), 500,1000,2000,3000,4000$ and 5000(c) from left to right. 
The first steady solution branch is solely depicted in Fig. 3(a). As seen in Fig. 3(a), the branch exists throughout the whole range of the Dean number in the present study. The branch starts from point a $(D n=0)$ and goes to the direction of increasing $D n$ as $\lambda$ decreases and extends up to point $c(D n=5000)$. To observe the change of the flow patterns and temperature distributions, contours of typical secondary flow and temperature profile at several Dean numbers are shown in Fig. 3(b), where the contours of $\psi$ and $T$ are drawn with the increments $\Delta \psi=0.6$ and $\Delta T=0.2$ respectively. The same increments of $\psi$ and $\mathrm{T}$ are used for all the figures in this paper, if not specified.

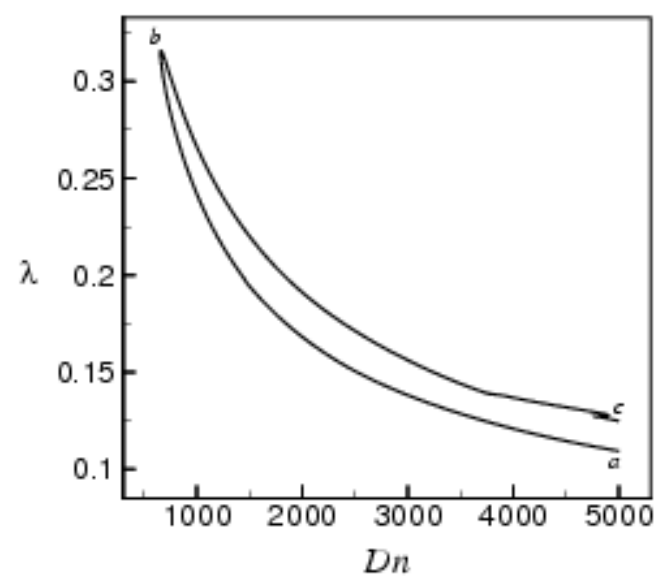

Figure 4: Second steady solution branch for $G r=100$ and $500 \leq D n \leq 5000$ at $\delta=0.1$.

The right-hand side of each duct box of $\psi$ and $\mathrm{T}$ is in the outside direction of the duct curvature. In the figures of the secondary flow, solid lines $\psi \geq 0$ show that the secondary flow is in the counter clockwise direction while the dotted lines $\psi<0$ in the clockwise direction. In the figures of the temperature field, solid lines are those for $T \geq 0$ and dotted ones for $T<0$. As seen in Fig. 3(b), the first steady solution branch consists of two-vortex solutions which are asymmetric with respect to the horizontal centre plane $y=0$. Heating the outer wall causes deformation of the secondary flow and yields asymmetry of the flow.

The second steady solution branch is shown in Fig. 4. As seen in Fig. 4, the branch starts from point $a(D n=5000)$ and goes to the direction of decreasing $D n$ as $\lambda$ increases and turns smoothly at point $b(D n=654.72)$ in the opposite direction. The branch then goes to the direction of increasing $D n$ as $\lambda$ decreases which extends up to point $d$ $(D n=5000)$. We show the secondary flow patterns and temperature profiles at several values of the Dean number on this branch in Fig. 5. It is found that the second branch starts with a two-vortex solution at point $a$ and becomes a four-vortex solution at point $b$ which remains a four-vortex solution up to point $c$. Thus we obtain a two-vortex solution on the lower branch, on the upper branch; however, we obtain four-vortex solutions. 
(a)
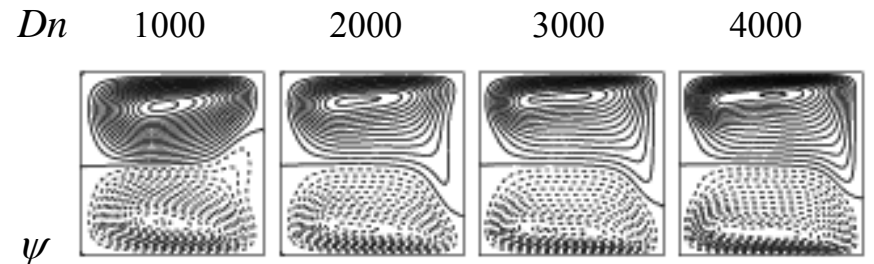

5000
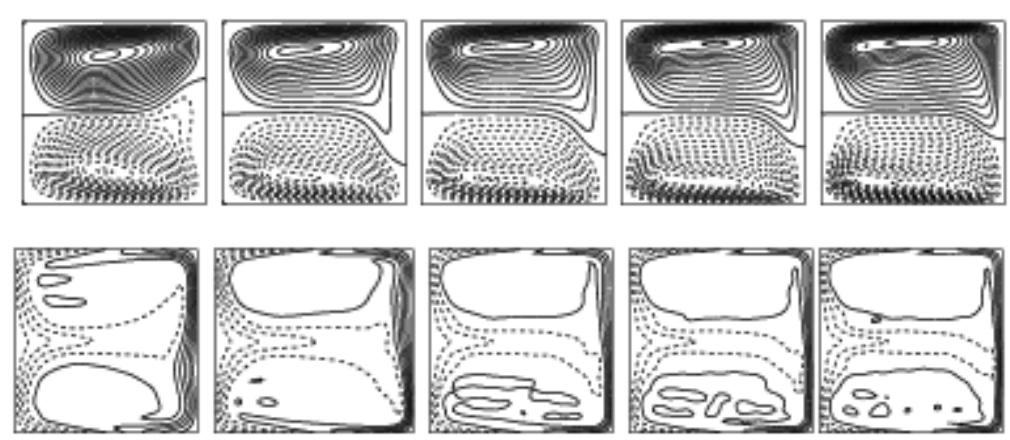

$T$
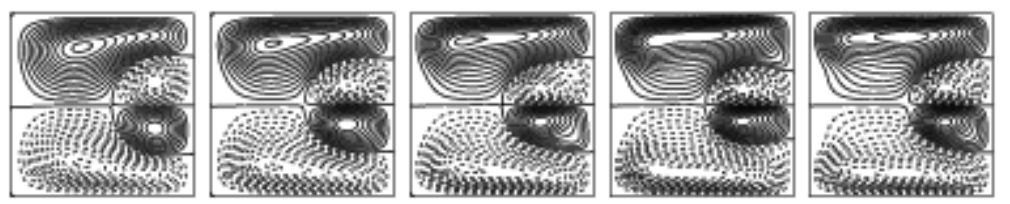

(b)
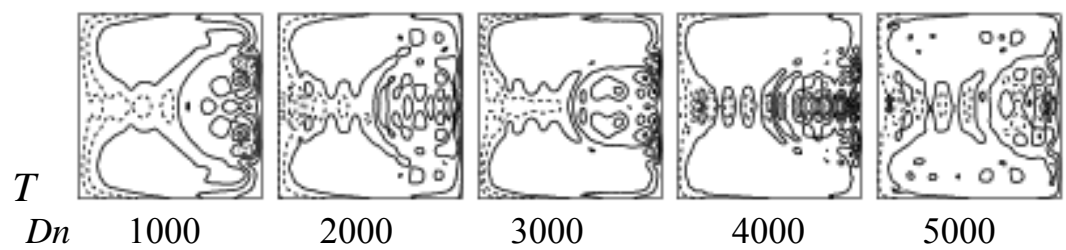

Figure 5: Contours of the secondary flow (top) and temperature profile (bottom) on the second steady solution branch for $\delta=0.1$ at $D n=1000,2000,3000,4000,5000$. (a) on the upper branch and (b) on the lower branch.

\subsection{Linear stability of the solutions}

In this paper, we examine linear stability of the steady solutions against only twodimensional ( $z$-independent) perturbations. To do this, the eigenvalue problem is solved which is constructed by the application of the function expansion method together with the collocation method to the perturbation equations obtained from Eqs. (2), (3) and (4). It is assumed that the time dependence of the perturbation is $e^{\sigma}$, where $\sigma=\sigma_{r}+i \sigma_{i}$ is the eigenvalue with $\sigma_{r}$ the real part, $\sigma_{i}$ the imaginary part and $i=\sqrt{-1}$. If all the real parts of the eigenvalue $\sigma$ are negative, the steady solution is linearly stable, but if there exists at least one positive real part of the eigenvalue, it is linearly unstable. In the unstable region, the perturbation grows monotonically for $\sigma_{i}=0$ and oscillatorily for $\sigma_{i} \neq 0$.

On the basis of the above-mentioned criteria, linear stability of the steady solutions is investigated. It is found that between the two steady solution branches; only the first branch is linearly stable in a couple of interval of $D n$, while the other branch is linearly unstable. The eigenvalues of the first steady solution branch are listed in Table 1 where the eigenvalues with the maximum real part of $\sigma$ are presented. Those for the linearly stable solutions are printed in bold letters. As seen in Table 1, the stability region exists for $0 \leq D n \leq 818.5$ and $1449.2 \leq D n \leq 2767.6$ and the perturbation grows oscillatorily 
$\left(\sigma_{i} \neq 0\right)$ for $818.6 \leq D n \leq 1449.1$ and $D n \geq 2767.7$. Therefore, the Hopf bifurcation occurs at $D n \approx 818.5$ and $D n \approx 2767.6$ and inverse Hopf bifurcation at $D n \approx 1449.2$. Linearly stable steady solution regions are shown with thick solid lines in Fig. 3(a).

Table 1: Linear stability of the first steady solution branch for $G r=100$ at $\delta=0.1$.

\begin{tabular}{cccc}
\hline$D n$ & $\lambda$ & $\sigma_{r}$ & $\sigma_{i}$ \\
\hline 0 & 0.000000 & $-8.379 \times 10^{-1}$ & 0 \\
100 & 1.031217 & -1.105 & 0 \\
500 & 0.360906 & -1.454 & 0 \\
818.5 & 0.269458 & $-5.461 \times 10^{-3}$ & \pm 8.078 \\
818.6 & 0.269440 & $3.288 \times 10^{-3}$ & \pm 8.088 \\
1200 & 0.219209 & 7.701 & $\pm 2.254 \times 10$ \\
1449.1 & 0.198380 & $1.389 \times 10^{-3}$ & $\pm 1.732 \times 10$ \\
1449.2 & 0.198373 & $-3.008 \times 10^{-3}$ & $\pm 1.731 \times 10$ \\
2000 & 0.169178 & -2.056 & 0 \\
2767.6 & 0.144324 & $-2.897 \times 10^{-3}$ & $\pm 1.074 \times 10^{2}$ \\
2767.7 & 0.144322 & $7.382 \times 10^{-4}$ & $\pm 1.075 \times 10^{2}$ \\
3500 & 0.129120 & $2.449 \times 10$ & $\pm 1.424 \times 10^{2}$ \\
4000 & 0.121279 & 3.473 & $\pm 1.623 \times 10^{2}$ \\
\hline
\end{tabular}

\subsection{Nusselt number}

We show the variation of the steady values of the Nusselt number with the Dean number in Fig. 6 for the first steady solution branch, where solid line denotes $N u_{c}$ on the cooled (inner) sidewall and dashed line $N u_{h}$ on the heated (outer) sidewall. Since between the two steady solution branches only the first branch is linearly stable, calculation of the steady values of the Nusselt number is meaningful only for this branch. Figure 6 shows that the tendency of increasing the Nusselt number is larger on the heated sidewall than that on the cooled sidewall for larger Dean numbers, which can be explained by the fact that many subsidiary secondary vortices are generated near the outer sidewall (Chandratilleke and Nursubyakto [15]). This result also suggests that heat transfer is significantly enhanced by the secondary flow from the heated wall to the fluid since the Nusselt number goes higher for the heated wall than that of the cooled wall, if the Dean number is increased. 


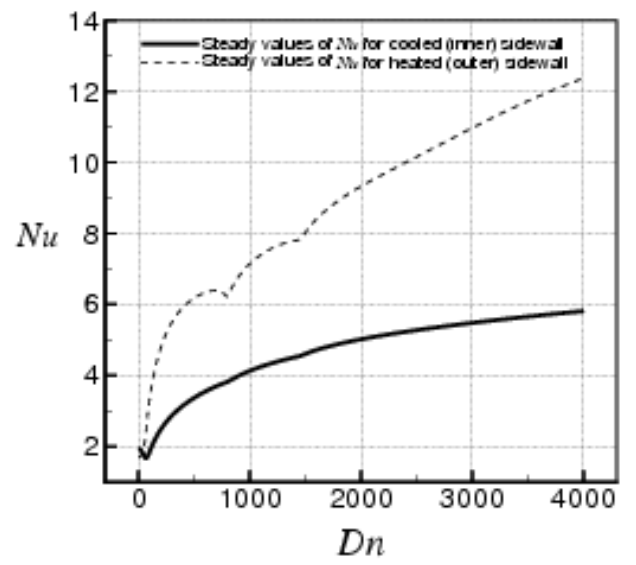

Figure 6: Steady values of the Nusselt number $(\mathrm{Nu})$ for the first steady solution branch.

\subsection{Time evolution}

In order to study the nonlinear behavior of the unsteady solutions, time evolution calculations of the Nusselt number are performed for both the cooled and heated sidewalls at several values of the Dean number. Figures 7 and 8 show the time evolution of $\mathrm{Nu}$ for the cooled $\left(N u_{c}\right)$ and heated $\left(N u_{h}\right)$ sidewalls, respectively.

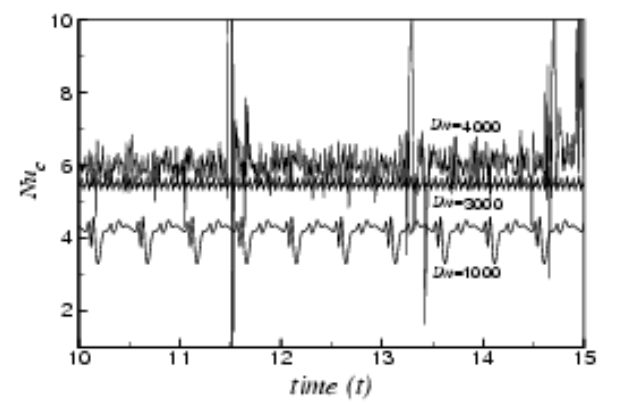

Figure 7: Time evolution of $N u$ at the cooled sidewall for $D n=1000,3000$ and 4000.

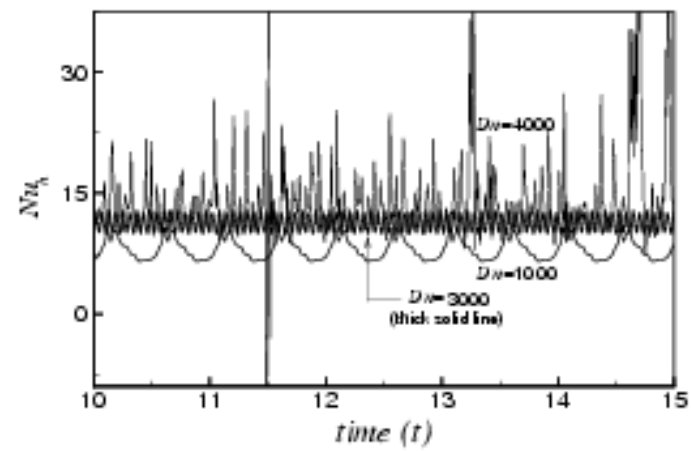

Figure 8: Time evolution of $N u$ at the heated sidewall for $D n=1000,3000$ and 4000.

Time evolutions of $N u$ for $D n \leq 818$, at which the steady solution is linearly stable on the first branch, show that the value of $\mathrm{Nu}$ quickly approaches that of the stable solution on 
the first branch. Then in order to see what happens when the steady solution is linearly unstable on the first branch for $819 \leq D n \leq 1449$, time evolution calculation is performed for both the sidewalls at $D n=1000$ as shown in Fig. 7. It is found that the unsteady solution is a periodic (or multi-periodic) oscillation at $D n=1000$. To observe the periodic change of the flow patterns and temperature distributions, contours of typical secondary flow and temperature profile are shown in Fig. 10, for one period of oscillation at $13.6 \leq t \leq 14.1$, where it is seen that the periodic (or multi-periodic) oscillation at $\mathrm{Dn}=1000$ is a two-vortex solution.

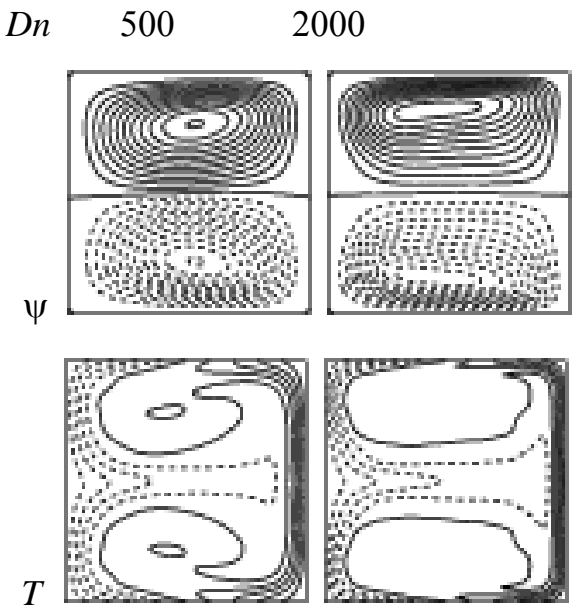

Figure 9: Contours of the secondary flow (top) and temperature profile (bottom) for one $\mathrm{Dn}=500$ and Dn $=2000$ at $t=10$.

Time evolution calculation is then performed for $1450 \leq D n \leq 2767$, where the steady solution is linearly stable on the first branch. It is found that the unsteady flow remains steady-state in this range whatever the initial condition we use. Figure 9 shows, for example, contours of secondary flow and temperature profile for $D n=500$ and 2000 at $t=10$, at which the steady flow is linearly stable in the first and second stable regions, respectively. Time evolutions of $N u$ are then performed for $D n \geq 2768$. Time evolutions of $N u$ for $D n=3000$ and 4000 are shown in Figs. 7 and 8 for the cooled and heated sidewalls, respectively. It is found that in the second unstable region, a periodic or multiperiodic oscillation occurs for $2768 \leq D n \leq 3570$; however, as $D n$ is increased further ( $D n=4000$ ), the periodic oscillation turns into a chaotic state. Then, in order to observe the change of the flow characteristics, as time proceeds, contours of secondary flow and temperature profile are shown in Figs. 11 and 12 for $D n=3000$ and 4000, respectively. As seen in Figs. 11 and 12, the periodic oscillation at $D n=3000$ is a two-vortex solution while aperiodic (chaotic) oscillation at $D n=4000$ is a two- and four-vortex solutions. In this regard, it should be worth mentioning that irregular oscillation of the flow through a curved duct has been observed experimentally by Ligrani and Niver [21] for the large aspect ratio. 


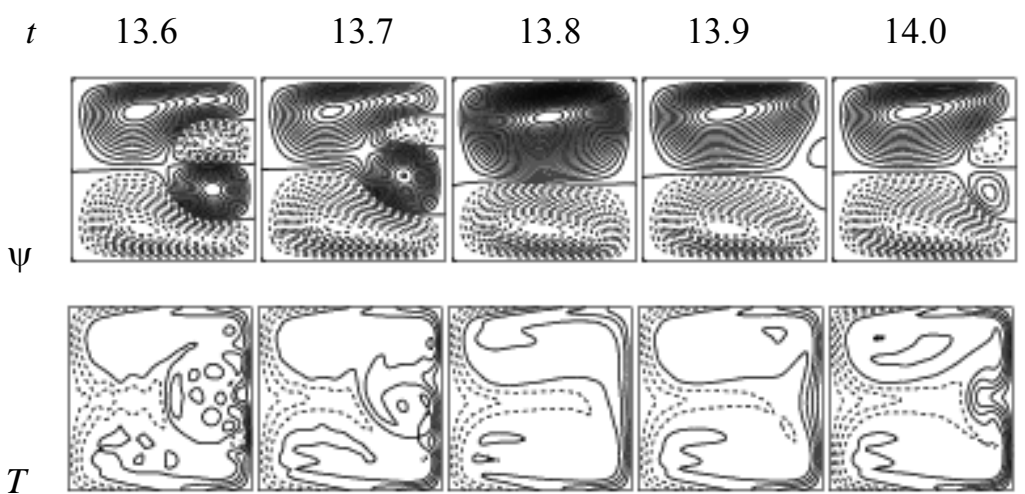

Figure 10: Contours of the secondary flow (top) and temperature profile (bottom) for one period of oscillation $13.6 \leq t \leq 14.1$ and $D n=1000$.

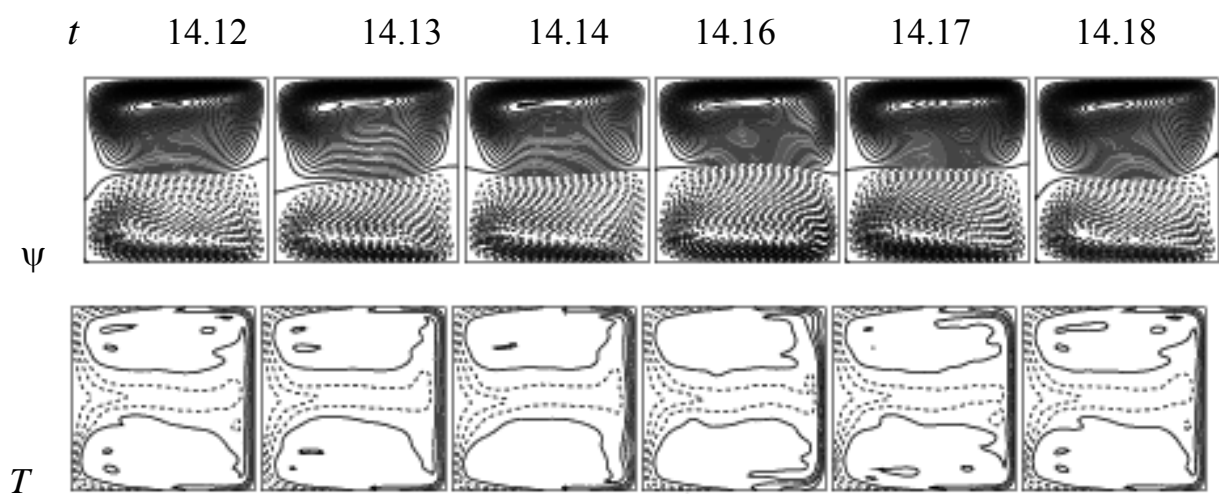

Figure 11: Contours of the secondary flow (top) and temperature profile (bottom) for one period of oscillation $14.12 \leq t \leq 14.18$ and $\mathrm{Dn}=3000$

$t$

12.0

12.2

12.4

12.6

12.8

13.0
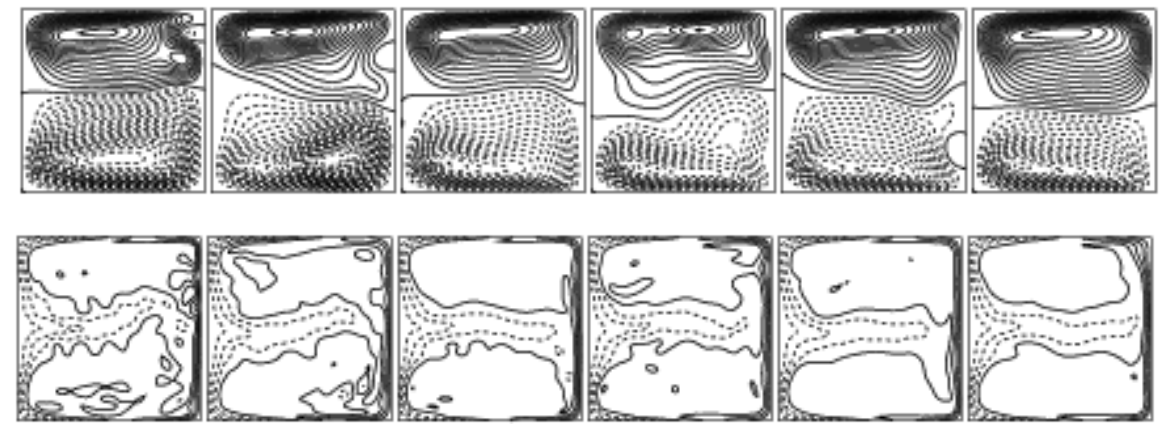

Figure 12: Contours of the secondary flow (top) and temperature profile (bottom) for one period of oscillation $12 \leq t \leq 13$ and $D n=4000$

By the time evolution calculations, it is found that stable steady solutions occur in the regions $0 \leq D n \leq 818$ and $1450 \leq D n \leq 2767$, periodic solutions for $819 \leq D n \leq 1449$ and $2768 \leq D n \leq 3570$ and chaotic solution for $D n \geq 3575$. Linear stability analysis indicates 
that the stable steady solution exists for $0 \leq D n \leq 818.5$ and $1449.2 \leq D n \leq 2767.6$. Therefore, the results of the linear stability analysis and those of the time evolution calculations are consistent.

\section{Conclusion}

A numerical study of the fully developed two-dimensional flow of viscous incompressible fluid through a curved duct of square cross section has been performed by using a spectral method and covering wide range of the Dean number for a fixed curvature $\delta=0.1$. In the present study, a temperature difference is applied across the vertical sidewalls for the Grashof number $G r=100$, where the outer wall is heated and the inner one cooled.

After a comprehensive survey over the range of the parameters, two branches of asymmetric steady solutions are obtained with two- and four-vortex solutions. Linear stability of the steady solutions reveals that only one branch is linearly stable in two distinct intervals of the Dean number $(\mathrm{Dn})$ while the other branch is completely unstable. It is found that the Hopf bifurcation occurs at the Dean numbers on the boundary between the stable and unstable Solutions. In order to investigate the transition from the multiperiodic oscillations to the chaotic states in more detail, the spectral analysis is found to be very useful. It is found that the chaotic solution is weak for small $D n$, where the solution drifts among the steady solution branches, for large $D n$, on the other hand, the chaotic solution becomes strong, where the solution gets away from the steady solution branches.

Steady values of the Nusselt numbers, $\mathrm{Nu}$, are also calculated for two differentially heated vertical sidewalls, and it is found that the larger the Dean number is, the larger the Nusselt number becomes on the outer sidewall. When there is no stable steady solution, time evolutions of $\mathrm{Nu}$ are calculated and it is found in the unstable region the flow undergoes through various flow instabilities, if the Dean number is increased.

\section{REFERENCES}

1. Dean, W. R., (1927). Note on the motion of fluid in a curved pipe. Philos. Mag., Vol. 4, pp. 208-223.

2. Berger, S. A., Talbot, L. and Yao, L. S., (1983). Flow in curved pipes, Annu. Rev. Fluid. Mech., Vol. 35, pp. 461-512.

3. Nandakumar, K. and Masliyah, J. H., (1986). Swirling flow and heat transfer in coiled and twisted pipes, Adv. Transport Process., Vol. 4, pp. 49-112

4. Ito, H., (1987). Flow in curved pipes, JSME Int. J., Vol. 30, pp. 543-552.

5. Dennis, S. C. R. and Ng, M., Dual solutions for steady laminar flow through a curved tube, Quart. J. Mech. Appl. Math., Vol. 35(1982), pp. 305-324.

6. Nandakumar K. and Masliyah, H. J., (1982). Bifurcation in steady laminar flow through curved tubes, J. Fluid Mech., Vol. 119, pp. 475-490.

7. Yanase, S., Goto, N. and Yamamoto, K., (1989). Dual solutions of the flow through a curved tube, Fluid Dyn. Res., Vol. 14, pp. 191-201.

8. Yang, Z. and Keller, H. B., (1986). Multiple laminar flows through curved pipes, Applied Numerical Mathematics, Vol. 2, pp. 257-271.

9. Thangam, S. and Hur, N., (1990). Laminar secondary flows in curved rectangular ducts, J. Fluid Mech., Vol. 217, pp. 421-440. 
10. Winters, K. H., (1987). A bifurcation study of laminar flow in a curved tube of rectangular cross-section, J. Fluid Mech., Vol. 180, pp. 343-369.

11. Yanase, S. and Nishiyama, K., (1988). On the bifurcation of laminar flows through a curved rectangular tube, J. Phys. Soc. Japan, Vol. 57, pp. 3790-3795.

12. Yanase, S., (1991). Time dependent analysis of bifurcating flows through a curved tube, Eur. J. Mech. B. Fluids, Vol. 10, pp. 321

13. Wang, L. and Yang, T., (2004). Bifurcation and stability of forced convection in curved ducts of square cross section, Int. J. Heat Mass Transfer, Vol. 47, pp. 2971-2987.

14. Wang, L. and Yang, T., (2005). Periodic oscillation in curved duct flows, Physica D, Vol. 200, pp. 296302.

15. Chandratilleke, T. T. and Nursubyakto, (2003). Numerical prediction of secondary flow and convective heat transfer in externally heated curved rectangular ducts, Int. J. Thermal Sci., Vol. 42, pp. 187-198.

16. Yanase, S., Mondal, R. N., Kaga, Y. and Yamamoto, K., (2005a). Transition from steady to chaotic states of isothermal and non-isothermal flows through a curved rectangular duct, J. Phys. Soc. Japan, Vol. 74, pp. 345-358.

17. Yanase, S., Mondal, R. N. and Kaga, Y., (2005b). Numerical study of non-isothermal flow with convective heat transfer in a curved rectangular duct, Int. J. Thermal Sci., Vol. 44, pp. 1047-1060.

18. Mondal, R. N., Kaga, Y., Hyakutake, T. and Yanase, S., (2007). Unsteady solutions and the bifurcation diagram for the flow through a curved square duct, Fluid Dyn. Res., Vol. 39, pp. 413-446

19. Mondal, R. N., Kaga, Y., Hyakutake, T. and Yanase, S., (2006). Effects of curvature and convective heat transfer in curved square duct flows, Trans. ASME, Journal of Fluids Engineering, Vol. 128, pp. 1013-1022.

20. Mondal, R. N., Isothermal and non-isothermal flows through curved ducts with square and rectangular cross sections, Ph.D. Thesis, Department of Mechanical Engineering, Okayama University, Japan, 2006.

21. Ligrani, P. M. and Niver, R. D., (1988). Flow visualization of Dean vortices in a curved channel with 40 to 1 aspect ratio, Phys. Fluids, Vol. 31, pp. 3605-3617. 